\title{
Upregulation of AGAT as a Possible Target in Molecular Nutrition
}

\author{
Sergej M. Ostojic \\ FSPE Applied Bioenergetics Lab, University of Novi Sad, Novi Sad, Serbia
}

\section{Dear Editor,}

L-arginine:glycine amidinotransferase (AGAT; EC 2.1.4.1) is a key enzyme in the biosynthesis of creatine, a natural amino acid derivative that plays a major role in temporal and spatial energy buffering. AGAT catalyzes the first and rate-limiting step in creatine synthesis, by transferring an amidino group from arginine to the $N^{\alpha}$ amine of glycine, to yield L-ornithine and guanidinoacetic acid (GAA). AGAT is a tissue-specific enzyme, predominantly expressed in the human kidney and pancreas, but also located in the liver, heart, brain, and testis. AGAT activity appears to be deficient in various medical conditions, including inherited cerebral creatine deficiency syndrome-3 (CCDS3, or AGAT deficiency), end-stage kidney disease, stroke, and vitamin E shortfall [1], all characterized by the low levels of creatine and clinical indices of impaired bioenergetics. For instance, circulating GAA and creatine levels, and renal AGAT activity were significantly lower in a chronic renal failure model, with elevated blood urea nitrogen suggestive of kidney damage [2]. Having this in mind, primary and secondary AGAT deficits thus highlight the enzyme as a sensible molecular target for various nutritional compounds aimed to upregulate its activity and recover energy metabolism.

A literature search, however, revealed an evident shortage of nutraceuticals capable to upregulate AGAT

karger@karger.com

(c) 2020 S. Karger AG, Basel

www.karger.com/anm

Karger! activity. While many nutritional agents are found to downregulate AGAT activity (e.g., creatine, L-arginine, GAA, ornithine) [1], only cysteine has been identified so far as a possible stimulator of AGAT function. Cysteine (2R-2-amino-3-sulfanylpropanoic acid) is a nonessential amino acid required for the synthesis of proteins and nonprotein compounds, including coenzyme A and glutathione. A seminal paper by Walker [3] evaluated the potential of various sulfhydryl compounds to modulate AGAT activity using in vitro hog and rat kidney samples. Cysteine appears to reverse the inhibition of AGAT (induced by L-canavanine and L-ornithine), with relative AGAT activity revived for $3 \%$ to 103 points (i.e., a relative activity of 100 represents $11 \mu \mathrm{moL}$ of arginine formed by the reaction). This perhaps suggests that cysteine instigates AGAT function, a feature that might have promising therapeutic potential in pathologies with mildly downregulated AGAT activity.

However, no clinical trials known to the author have evaluated if dietary cysteine induces AGAT stimulation. A recent double-blind placebo-controlled crossover trial has found that a single dose of cysteine derivative $(2,400$ mg of $\mathrm{N}$-acetyl-L-cysteine, NAC) improved biomarkers of brain creatine metabolism (e.g., glutamate/glutamineto-creatine ratio) in patients with schizophrenia [4]. These data provide preliminary evidence that NAC mod- 
ulates human brain bioenergetics, and AGAT upregulation might be involved. More studies are needed to validate the possible role of dietary cysteine in AGAT upregulation in a clinical environment, accounting for organ-specific responses and different administration protocols, also to discover and scrutinize other nutraceuticals that might stimulate AGAT, a worthwhile target in molecular nutrition and biomedicine.

\section{Disclosure Statement}

The author has no conflicts of interest to disclose.

\section{Funding Sources}

No funding was received.

\section{References}

1 Wyss M, Kaddurah-Daouk R. Creatine and creatinine metabolism. Physiol Rev. 2000 Jul; 80(3):1107-213.

2 Kuroda M. Study on impaired metabolism of guanidinoacetic acid in chronic renal failure rabbits with special reference to impaired conversion of arginine to guanidinoacetic acid. Nephron. 1993;65(4):605-11.
3 Walker JB. Studies on the mechanism of action of kidney transamidinase. J Biol Chem. 1957 Jan;224(1):57-66.

4 McQueen G, Lally J, Collier T, Zelaya F, Lythgoe DJ, Barker GJ, et al. Effects of N-acetylcysteine on brain glutamate levels and resting perfusion in schizophrenia. Psychopharmacology (Berl). 2018 Oct;235(10):3045-54. 